\title{
Coherent quantum transport in the presence of a finite-range transversely polarized time-dependent field
}

\author{
C. S. Tang ${ }^{1}$ and C. S. $\mathrm{Chu}^{2}$ \\ ${ }^{1}$ Physics Division, National Center for Theoretical Sciences, P.O. Box 2-131, Hsinchu 30013, Taiwan \\ ${ }^{2}$ Department of Electrophysics, National Chiao Tung University, Hsinchu 30010, Taiwan
}

\begin{abstract}
This work investigates the quantum transport in a narrow constriction acted upon by a finiterange transversely polarized time-dependent electric field. A generalized scattering-matrix method is developed that has incorporated a time-dependent mode-matching scheme. The transverse field induces coherent inelastic scatterings that include both intersubband and intersideband transitions. These scatterings give rise to the dc conductance $G$ a general suppressed feature that escalates with the chemical potential. In addition, particular suppressed features - the dip structures - are found in $G$. These features are recognized as the quasi-bound-state (QBS) features that arise from electrons making intersubband transitions to the vicinity of a subband bottom. For the case of larger field intensities, the QBS features that involve more photons are more evident. These QBS features are closely associated with the singular density of states at the subband bottoms. An experimental setup is proposed for the observation of these features.
\end{abstract}

PACS numbers: 73.23.-b, 72.10.-d, 72.40.+w

\section{INTRODUCTION}

Advances in the epitaxial growth technologies have lead to the fabrication of high-quality two-dimensional electron gas (2DEG) systems that are almost defect-free and upon which electronic nanostructures can be built. The electron transport properties of these nanostructures have been studied extensively both experimentally and theoretically 1.2 The most studied nanostructure is the quantum point contact (QPC), due to its simple configuration, and due also to the significant quantization effects in such systems, as is shown in the conductance $G .3 .4 .5$

These QPC's, when created electrostatically by negatively biasing a split-gate located on top of a $2 \mathrm{DEG}, 3,4,5$ can be pictured as a narrow constriction connecting adiabatically at each end to a $2 \mathrm{DEG}, \stackrel{6.7}{a}$ as depicted in Fig. 1] The energy levels in the narrow constriction are quantized into one-dimensional subbands which density of states (DOS) is singular near a subband bottom. This singular DOS was found, in the presence of an attractive scatterer, to give rise to dip structures in $G, \frac{8.9,10,11,12.13 .14}{1}$ which is associated with the formation of impurity-induced QBS's 9 formed just beneath a sub-

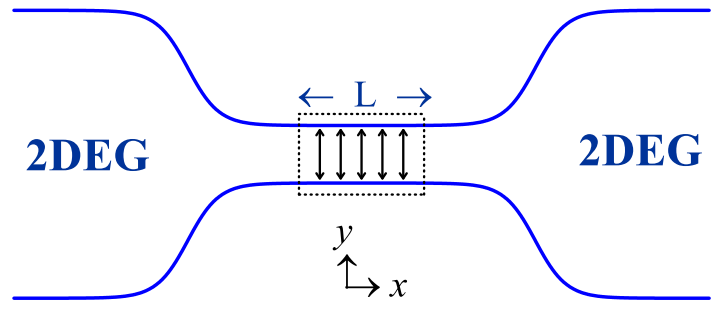

FIG. 1: Sketch of the gated QPC which is connected at each end to a two-dimensional electron gas electrode. The narrow constriction is acted upon by external transversely polarized time-dependent electric field within millimeter wave regime. band bottom.

More recently, attentions have been shifted to QPC's acted upon by high frequency fields. These time-dependent fields include transversely, 15.16 .17 .18 .19 .20 .21 .22 .23 .24 .25 .26 .27 .28 or longitudinally ${ }^{29.30}$ polarized fields, or simply gate-induced time-modulated potentials 33.34 .35 These studies focus on coherent inelastic scatterings by assuming the range of the time-modulated fields to be shorter than the incoherent mean free path. A number of interesting transport characteristics were explored. In the case of zero source-drain bias, it was demonstrated that electron pumping could occur when an asymmetric spit-gate is acted upon by a time-modulated field. 15 In the case of a finite source-drain bias, and the QPC's have varying widths, the photon-assisted quantum transport characteristics have been studied 16.17.18.19.20.21.22.23.24.25 One might also be prompted by the impurity-induced QBS features $, 8.9,10,11,12,13,14$ and ask whether the QBS's could find their way of manifestation in the time-dependent transport characteristics in QPC's. Indeed, earlier studies have already found QBS features for cases when the QPC's have either a delta-profile oscillating barrier ${ }^{33}$ or transverse field ${ }^{26}$ In a recent study, we consider the more realistic case of a finite-range longitudinally polarized field, 30 and we find QBS features that are associated with electrons making intrasubband and intersideband transitions to the vicinity of a subband bottom. Since the transitions allowed depend on the polarization of the time-modulated field, we opt to investigate in this paper the QBS features for a finite-range transversely polarized field.

In theoretical studies of coherent quantum transport in mesoscopic nanostructures, the transfer-matrix and the scattering-matrix method are powerful tools. Both the methods enable us to numerically calculate current transmission probabilities for arbitrary shaped configurations. The conductance of the nanostructure can be obtained 
by Landauer-Büttiker formalism $\stackrel{31.32}{ }$ However, since the transfer-matrix method fails in systems for which energy conservation is violated, it is unapt for a time-dependent external field acting upon the system. Thus, one has to develop a generalized scattering-matrix method to calculate numerically the current transmission probability of a time-modulated mesoscopic nanostructure.

In this paper, we develop a generalized scattering method for a finite-range transversely polarized timedependent electric field acting upon a narrow constriction, as depicted in Fig. 1] This method enables us to calculate not only the current transmission and reflection probabilities but also the contributions from each subband and sideband states yielded by the transversely polarized electric field. This transverse field $\mathbf{E}(\mathbf{x}, t)=$ $\mathcal{E}(x) \cos (\omega t) \hat{y}$ has a finite longitudinal profile, namely that $\mathcal{E}(x)$ covers a length $L$ that excludes the reservoirs. The finiteness of the electric field in the $x$ direction breaks the longitudinal translational invariance, and hence allows electrons to make intersideband transitions not to conserve their longitudinal momenta ${ }^{26.34}$ Moreover, since the transverse electric field is not uniform in the $y$ direction, the transverse translational invariance is also violated. Thus the electron-photon scattering processes can include intersubband transitions. This transmission mechanism is quite different with the scatterings that induced by a longitudinal field $\underline{\underline{30}}$ or a time-modulated potential, ${ }^{34}$ where the electrons can only make intrasubband transitions (the subband index remains unchanged).

The rest of this paper is organized as follows. In Sec. II, the generalized scattering-matrix method is formulated that has incorporated a time-dependent mode-matching scheme to solve the time-dependent Schrödinger equation. The method described in Sec. II is calculated numerically in Sec. III. From our numerical evidence, we predict that QBS features caused by the transversely polarized field can occur in narrow constrictions. Concluding remarks are given in Sec. IV.

\section{MODEL AND METHOD}

In this section, the coherent inelastic scattering problem in the presence of a finite-range transverse electric field is formulated. The finite-range time-dependent electric field is divided by a series of segments, each of them is described by a $\delta$-profile field ${ }^{26}$ The matching between these sliced regions has to be performed in the cascading of the scattering matrices, from which the transmission and reflection coefficients are obtained. The conductance $G$ is then expressed in terms of these coefficients.

Previously, we have investigated transport properties of electrons in narrow constrictions acted upon by a longitudinally polarized time-dependent electric field ${ }^{30}$ The potential due to the longitudinal field that have a finite range in the $x$ direction, but remain uniform in the $y$ direction. This uniformity in the transverse direction allows us to propose a matching scheme that avoids slicing the region covered by the longitudinal time-dependent field. However, as long as the narrow constriction is acted upon by a transversely polarized time-dependent field, the translational invariance in the $y$ direction is breaked - both intersubband and intersideband transitions are involved. Hence, we have to develop a generalized scattering method to formulate the quantum transport problem when a transverse external field acts upon the narrow constriction.

Since the electric field is assumed to be applied only on the narrow constriction region, we need only to formulate this scattering problem in the narrow constriction region. In the ballistic regime, the length of the narrow constriction, $L_{c}$, is smaller than the phase breaking length, $l_{\phi}$, and hence the electron transport can be treated as a single particle problem. Thus the electron transport can be formulated by a time-dependent Schrödinger equation, given by

$$
i \hbar \frac{\partial}{\partial t} \Psi(\mathbf{x}, t)=\mathcal{H}(\mathbf{x}, t) \Psi(\mathbf{x}, t),
$$

with the Hamiltonian of the form

$$
\mathcal{H}(\mathbf{x}, t)=\left[\mathbf{p}+\frac{e}{c} \mathbf{A}(\mathbf{x}, t)\right]^{2}+V_{c}(y) .
$$

Here $\mathbf{p}$ denotes the momentum of an electron and $V_{c}(y)$ represents the transverse confinement of the narrow constriction modeled by a quadratic potential ${ }^{36}$ Taking the Coulomb gauge, the effect of the transversely polarized electric field can be represented by a vector potential:

$$
\mathbf{A}(\mathbf{x}, t)=-\frac{c}{\omega} \mathcal{E}(x) \sin (\omega t) \hat{y},
$$

where $\mathcal{E}(x)$ represents the profile of the external field with amplitude $\mathcal{E}_{0}$ for $|x|<L / 2$ and vanishes otherwise.

To be convenient for analysis, below we choose the length unit $a^{*}=1 / k_{\mathrm{F}}$, the energy unit $E^{*}=\hbar^{2} k_{\mathrm{F}}^{2} /\left(2 m^{*}\right)$, the time unit $t^{*}=\hbar / E^{*}$, and field amplitude $\mathcal{E}_{0}$ in units of $E^{*} /\left(e a^{*}\right)$, where $-e$ denotes the electron charge, with effective mass $m^{*}$, and $k_{\mathrm{F}}$ represents a typical Fermi wave vector of the reservoir. Thus we can write the dimensionless transverse confinement $V_{c}(y)=\omega_{y}^{2} y^{2}$, and then gives the quantized transverse energy levels $\varepsilon_{n}=(2 n+1) \omega_{y}$ and the corresponding wave function $\phi_{n}(y)$.

The finite-range electric field is divided by $N_{L}$ slices, thus the width of every slice is given by $\delta L=L / N_{L}$. Here the sufficiently large amount of $N_{L}$ is needed such that $\delta L$ is sufficiently narrow to ensure every slice can be described by a $\delta$-profile field ${ }^{26}$ The locations of these $\delta$-profile fields are given by $x_{i}=-L / 2+(i-1 / 2) \delta L$, where the positive integer $i=1,2, \cdots, N_{L}$. By dividing the profile slice-wisely, the Schrödinger equation of the $i$ th $\delta$-profile field is then given by 


$$
i \frac{\partial}{\partial t} \Phi^{(i)}(\mathbf{x}, t)=\left[-\left(\frac{\partial^{2}}{\partial x^{2}}+\frac{\partial^{2}}{\partial y^{2}}\right)+\omega_{y}^{2} y^{2}+\left(i \frac{2 \mathcal{E}_{0}}{\omega} \frac{\partial}{\partial y} \sin (\omega t)+\frac{\mathcal{E}_{0}^{2}}{\omega^{2}} \sin ^{2}(\omega t)\right) \delta L \delta\left(x-x_{i}\right)\right] \Phi^{(i)}(\mathbf{x}, t) .
$$

Considering a $n$th subband electron incident from left-hand side of the $i$ th $\delta$-profile field, and with incident energy $\mu^{\prime}$, the scattering wave function is given by ${ }^{26}$

$$
\begin{aligned}
\Phi_{n}^{(i)}(\mathbf{x}, t)= & \phi_{n}(y) \exp \left[i k_{n}\left(\mu^{\prime}\right) x-i \mu^{\prime} t\right] \\
& +\sum_{n^{\prime}, m^{\prime}} r_{n^{\prime} n}^{(i)}\left(m^{\prime}\right) \phi_{n^{\prime}}(y) \exp \left[-i k_{n^{\prime}}\left(\mu^{\prime}+m^{\prime} \omega\right) x\right] \exp \left[-i\left(\mu^{\prime}+m^{\prime} \omega\right) t\right] \quad \text { if } x<x_{i}, \\
\Phi_{n}^{(i)}(\mathbf{x}, t)= & \sum_{n^{\prime}, m^{\prime}} t_{n^{\prime} n}^{(i)}\left(m^{\prime}\right) \phi_{n^{\prime}}(y) \exp \left[i k_{n^{\prime}}\left(\mu^{\prime}+m^{\prime} \omega\right) x\right] \exp \left[-i\left(\mu^{\prime}+m^{\prime} \omega\right) t\right] \quad \text { if } x>x_{i},
\end{aligned}
$$

where the electron is scattered into the subband $n^{\prime}$ and sideband $m^{\prime}$. The wave vector $k_{n^{\prime}}\left(\mu^{\prime}\right)=\sqrt{\mu^{\prime}-\varepsilon_{n}}$ is the effective wave vector for the electron with energy $\mu^{\prime}$ and in the $n$th subband. Here we have defined $\Phi^{(i)}(\mathbf{x}, t)=$ $\sum_{n} \Phi_{n}^{(i)}(\mathbf{x}, t)$ as a summation over all occupied incident subbands. The coefficients in Eq. (5) and (6) have to be determined by the following boundary conditions:

$$
\left.\Phi_{n}^{(i)}\right|_{x=x_{i}-\delta}=\left.\Phi_{n}^{(i)}\right|_{x=x_{i}+\delta}
$$

and

$$
\begin{aligned}
& \left.\frac{\partial \Phi_{n}^{(i)}}{\partial x}\right|_{x=x_{i}+\delta}-\left.\frac{\partial \Phi_{n}^{(i)}}{\partial x}\right|_{x=x_{i}-\delta} \\
= & {\left[i \frac{2 \mathcal{E}_{0}}{\omega} \frac{\partial}{\partial y} \sin (\omega t)+\frac{\mathcal{E}_{0}^{2}}{\omega^{2}} \sin ^{2}(\omega t)\right] \delta L \Phi_{n}^{(i)}\left(x=x_{i}\right) . }
\end{aligned}
$$

Imposing the boundary conditions (7) and (9) to perform the matching at all times and given the expression of the matrix element

$$
<l\left|\frac{\partial}{\partial y}\right| n^{\prime}>=\sqrt{\frac{\omega_{y}}{2}}\left[\sqrt{n^{\prime}} \delta_{l, n^{\prime}-1}-\sqrt{n^{\prime}+1} \delta_{l, n^{\prime}+1}\right]
$$

we obtain the equations relating the reflection coefficients $r_{l n}^{(i)}(m)$ and the transmission coefficients $t_{l n}^{(i)}(m)$,

$$
t_{l n}^{(i)}(m)-r_{l n}^{(i)}(m)=\delta_{m, 0} \delta_{n, l},
$$

and

$$
\begin{gathered}
\delta_{m, 0} \delta_{n, l} k_{n}\left(\mu^{\prime}\right) \\
=k_{l}\left(\mu^{\prime}+m \omega\right)\left[r_{l n}^{(i)}(m)+t_{l n}^{(i)}(m)\right] \\
+i \frac{\mathcal{E}_{0}}{\omega} \delta L \sum_{n^{\prime}, m^{\prime}}\left[\delta_{m^{\prime}, m+1}-\delta_{m^{\prime}, m-1}\right] \\
\quad \times<l\left|\frac{\partial}{\partial y}\right| n^{\prime}>t_{n^{\prime} n}^{(i)}\left(m^{\prime}\right) \\
+i \frac{\mathcal{E}_{0}^{2}}{4 \omega^{2}} \delta L\left[2 t_{l n}^{(i)}(m)+t_{l n}^{(i)}(m+2)+t_{l n}^{(i)}(m-2)\right] .
\end{gathered}
$$

and

$$
\widetilde{r}_{l n}^{(i)}(m)=r_{l n}^{(i)}(m) \exp \left\{-2 i\left[k_{l}\left(\mu^{\prime}+m \omega\right)+k_{n}\left(\mu^{\prime}\right)\right] x_{i}\right\} .
$$

In general, for an electron incident from the left-hand side of the $i$ th slice (the $(i-1)$ th region) in the subband $n_{i-1}$ and at energy $\mu+m_{i-1} \omega$, this incident state is denoted as $\alpha_{i-1}=\left(n_{i-1}, m_{i-1}\right)$. The electron may be transmitted to the right-hand side of the $i$ th slice ( $i$ th region) into the state $\alpha_{i}=\left(n_{i}, m_{i}\right)$ with a transmission coefficient $t_{\alpha_{i}, \alpha_{i-1}}$. Also, the electron may be reflected to the left-hand side of the $i$ th slice $((i-1)$ th region $)$ into the state $\beta_{i-1}=\left(n_{i-1}^{\prime}, m_{i-1}^{\prime}\right)$ with a reflection coefficient $r_{\beta_{i-1}, \alpha_{i-1}}$. Similarly, for an electron incident from th right-hand side of the $i$ th slice in the incident state $\beta_{i}=\left(n_{i}^{\prime}, m_{i}^{\prime}\right)$, the corresponding transmission coefficient and reflection coefficient due to this slice are given by $\widetilde{t}_{\beta_{i-1}, \beta_{i}}$ and $\widetilde{r}_{\beta_{i-1}, \beta_{i}}$, respectively. After defining these 


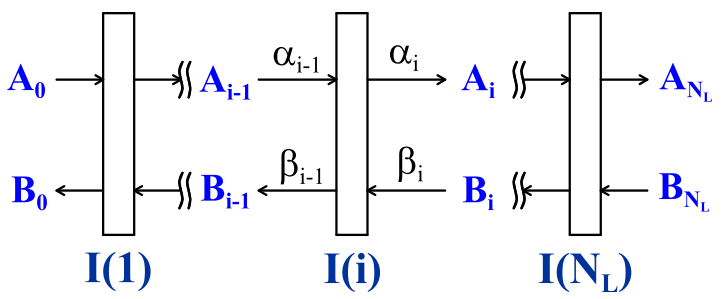

FIG. 2: Sketch of the finite-range trnasverse electric field, which is divided into $N_{L}$ slices. Every slice is described by a $\delta$-profile field, and it is between the $(i-1)$ th and the $i$ th region. The coefficients of the right and left going states of the $i$ th region are denoted as $\mathbf{A}_{i}$ and $\mathbf{B}_{i}$, respectively, and these coefficients between successive regions are connected by the interface matrix $\mathbf{I}(i) . \alpha_{i}$ and $\beta_{i}$ are dummy indices of the $i$ th region, including both subband and sideband indices.

coefficients, we can establish the scattering matrix equation, given by

$$
\left[\begin{array}{c}
\mathbf{A}_{i} \\
\mathbf{B}_{i-1}
\end{array}\right]=\mathbf{S}(i-1, i)\left[\begin{array}{c}
\mathbf{A}_{i-1} \\
\mathbf{B}_{i}
\end{array}\right]
$$

where $\mathbf{A}_{i}$ and $\mathbf{B}_{i}$ are the coefficients of the right- and the left-going states in the $i$ th region, respectively, as illustrated in Fig. 2 Here $\mathbf{S}(i-1, i)$ is the scattering matrix which connects the $(i-1)$ th to the $i$ th region, across the $i$ th slice, given by

$$
\mathbf{S}(i-1, i)=\left[\begin{array}{cc}
\mathbf{t}(i) & \widetilde{\mathbf{r}}(i) \\
\mathbf{r}(i) & \widetilde{\mathbf{t}}(i)
\end{array}\right] .
$$

Here, $\mathbf{t}(i)$ and $\mathbf{r}(i)$ denote the transmission and reflection matrices of the right-going electron at the $i$ th slice, respectively, and the tilded two refer to the contribution of left-going electron. Matching between these sliced regions has to be performed in the cascading of the scattering matrices. We should point out the reason for using the scattering matrix formalism, instead of the transfer matrix method, is to avoid the use of truncation schemes required in dealing with the exponentially growing solutions. The scattering matrix method is stable and accu- rate without any special treatment for any of the intermediate states.

To do the piece-wise matching, we start from rearranging Eq. (14) to obtain the following matrix equation

$$
\left[\begin{array}{l}
\mathbf{A}_{i-1} \\
\mathbf{B}_{i-1}
\end{array}\right]=\mathbf{I}(i)\left[\begin{array}{l}
\mathbf{A}_{i} \\
\mathbf{B}_{i}
\end{array}\right]
$$

which connects the coefficients of the successive regions across the $i$ th slice. Here $\mathbf{I}(i)$ is the interface matrix of the $i$ th slice, defined by

in which

$$
\mathbf{I}(i)=\left[\begin{array}{ll}
\mathbf{I}_{11}(i) & \mathbf{I}_{12}(i) \\
\mathbf{I}_{21}(i) & \mathbf{I}_{22}(i)
\end{array}\right],
$$

$$
\begin{aligned}
& \mathbf{I}_{11}(i)=\mathbf{t}(i)^{-1}, \\
& \mathbf{I}_{12}(i)=-\mathbf{t}(i)^{-1} \widetilde{\mathbf{r}}(i), \\
& \mathbf{I}_{21}(i)=\mathbf{r}(i) \mathbf{t}(i)^{-1}, \\
& \mathbf{I}_{22}(i)=\widetilde{\mathbf{t}}(i)-\mathbf{r}(i) \mathbf{t}(i)^{-1} \widetilde{\mathbf{r}}(i) .
\end{aligned}
$$

In general, for the regions up to the $(i-1)$ th slice, we have

$$
\left[\begin{array}{c}
\mathbf{A}_{i-1} \\
\mathbf{B}_{0}
\end{array}\right]=\mathbf{S}(0, i-1)\left[\begin{array}{c}
\mathbf{A}_{0} \\
\mathbf{B}_{i-1}
\end{array}\right]
$$

where $\mathbf{S}(0, i-1)$ is the scattering matrix connecting the 0th region to the $(i-1)$ th region, defined by

$$
\mathbf{S}(0, i-1)=\left[\begin{array}{ll}
\mathbf{S}_{11}(0, i-1) & \mathbf{S}_{12}(0, i-1) \\
\mathbf{S}_{21}(0, i-1) & \mathbf{S}_{22}(0, i-1)
\end{array}\right] .
$$

Imposing Eqs. (16) and (19), the coefficients $\mathbf{A}_{i-1}$ and $\mathbf{B}_{i-1}$ may be eliminated, and then we obtain the matrix equation connecting the 0 th to the $i$ th region, given by

$$
\left[\begin{array}{l}
\mathbf{A}_{i} \\
\mathbf{B}_{0}
\end{array}\right]=\mathbf{S}(0, i)\left[\begin{array}{l}
\mathbf{A}_{0} \\
\mathbf{B}_{i}
\end{array}\right] \text {. }
$$

The submatrices of this scattering matrix $\mathbf{S}(0, i)$ are, explicitly 38

$$
\begin{aligned}
& \mathbf{S}_{11}(0, i)=\left[\mathbf{I}_{11}(i)-\mathbf{S}_{12}(0, i-1) \mathbf{I}_{21}(i)\right]^{-1} \mathbf{S}_{11}(0, i-1), \\
& \mathbf{S}_{12}(0, i)=\left[\mathbf{I}_{11}(i)-\mathbf{S}_{12}(0, i-1) \mathbf{I}_{21}(i)\right]^{-1}\left[\mathbf{S}_{12}(0, i-1) \mathbf{I}_{22}(i)-\mathbf{I}_{12}(i)\right], \\
& \mathbf{S}_{21}(0, i)=\mathbf{S}_{21}(0, i-1)+\mathbf{S}_{22}(0, i-1) \mathbf{I}_{21}(i) \mathbf{S}_{11}(0, i), \\
& \mathbf{S}_{22}(0, i)=\mathbf{S}_{22}(0, i-1) \mathbf{I}_{22}(i)+\mathbf{S}_{22}(0, i-1) \mathbf{I}_{21}(i) \mathbf{S}_{12}(0, i) .
\end{aligned}
$$

This iterative procedure is not as easy to evaluate in terms of the transfer-matrix method, which simply inverses a product of matrices. More precisely, once the system is acted upon by an external time-modulated field, the evanescent modes play an important role due to inelastic scatterings. In this situation, we may prefer to use the scattering-matrix method to gain the stability for the numerical computation. 
By iterating Eq. (22), we obtain the scattering matrix $\mathbf{S}\left(0, N_{L}\right)$ which satisfies the matrix equation:

$$
\left[\begin{array}{c}
\mathbf{A}_{N_{L}} \\
\mathbf{B}_{0}
\end{array}\right]=\mathbf{S}\left(0, N_{L}\right)\left[\begin{array}{c}
\mathbf{A}_{0} \\
\mathbf{B}_{N_{L}}
\end{array}\right] \text {. }
$$

This equation describes the electron transport through the whole time-modulated region. The incident state is assumed to be $\alpha_{\text {in }}=\left(n_{0}, 0\right)$ such that the elements of the incident coefficient $\mathbf{A}_{0}$ can be expressed as $\delta_{n, n_{0}} \delta_{m, 0}$, i.e., only $\left(n_{0}, 0\right)$ is the nonzero element. Setting $\mathbf{B}_{N_{L}}=\mathbf{0}$, we have

$$
\mathbf{A}_{N_{L}}=\mathbf{S}_{11}\left(0, N_{L}\right) \mathbf{A}_{0},
$$

and

$$
\mathbf{B}_{0}=\mathbf{S}_{21}\left(0, N_{L}\right) \mathbf{A}_{0} .
$$

The transmission coefficient for an electron incident from the initial state $\alpha_{\mathrm{in}}=\left(n_{0}, 0\right)$ and transmitted, by the finite-range transverse field, into the final state $\alpha_{\mathrm{f}}=$ $\left(n_{\mathrm{f}}, m_{\mathrm{f}}\right)$ is denoted by $t_{\alpha_{\mathrm{f}}, \alpha_{\mathrm{in}}}=\left(A_{N_{L}}\right)_{\alpha_{\mathrm{f}}}$, where $\left(A_{N_{L}}\right)_{\alpha_{\mathrm{f}}}$ is an element of $\mathbf{A}_{N_{L}}$. The current transmission coefficient, corresponding to this inelastic scattering process, is then given by

$$
T_{\alpha_{\mathrm{in}}}^{\alpha_{\mathrm{f}}}=\left[\frac{k_{n_{\mathrm{f}}}\left(\mu+m_{\mathrm{f}} \omega\right)}{k_{n_{0}}(\mu)}\right]\left|t_{\alpha_{\mathrm{f}}, \alpha_{\mathrm{in}}}\right|^{2} .
$$

Therefore, the zero temperature conductance may obtained by summing over all the possible incident and transmitted states, given by

$$
G=\frac{2 e^{2}}{h} \sum_{\alpha_{\mathrm{in}}} \sum_{\alpha_{\mathrm{f}}} T_{\alpha_{\mathrm{in}}}^{\alpha_{\mathrm{f}}}=\frac{2 e^{2}}{h} \sum_{\alpha_{\mathrm{in}}} T_{\alpha_{\mathrm{in}}},
$$

where $T_{\alpha_{\text {in }}}$ is the current transmission coefficient from the incident state $\alpha_{\text {in }}$. Since the incident sideband is specified to be $m=0$ for an arbitrary incident subband $n_{0}$, so that the summation $\sum_{\alpha_{\text {in }}}=\sum_{n_{0}=0}^{N}$, where $N+1$ is the number of propagating subbands for the chemical potential $\mu$. But for the final states, both the subband and sideband indices are arbitrary, thus $\sum_{\alpha_{\mathrm{f}}}=\sum_{n_{\mathrm{f}}=0}^{N} \sum_{m_{\mathrm{f}}}^{\prime}$ is expected to be a double sum. Here the superscript prime indicates that summation is over $m_{\mathrm{f}}$ such that $k_{n_{\mathrm{f}}}\left(\mu+m_{\mathrm{f}} \omega\right)$ is real, namely that only occupied subbands are included for the scattering states. The conservation of current, given by the condition

$$
\sum_{\alpha_{\mathrm{f}}} \frac{k_{n_{\mathrm{f}}}\left(\mu+m_{\mathrm{f}} \omega\right)}{k_{n_{0}}(\mu)}\left[\left|t_{\alpha_{\mathrm{f}}, \alpha_{\text {in }}}\right|^{2}+\left|r_{\alpha_{\mathrm{f}}, \alpha_{\text {in }}}\right|^{2}\right]=1 .
$$

is used to check our numerical accuracy.

\section{NUMERICAL RESULTS AND DISCUSSION}

In this section the behavior of the conductance $G$ is studied. To facilitate the experimental performance we

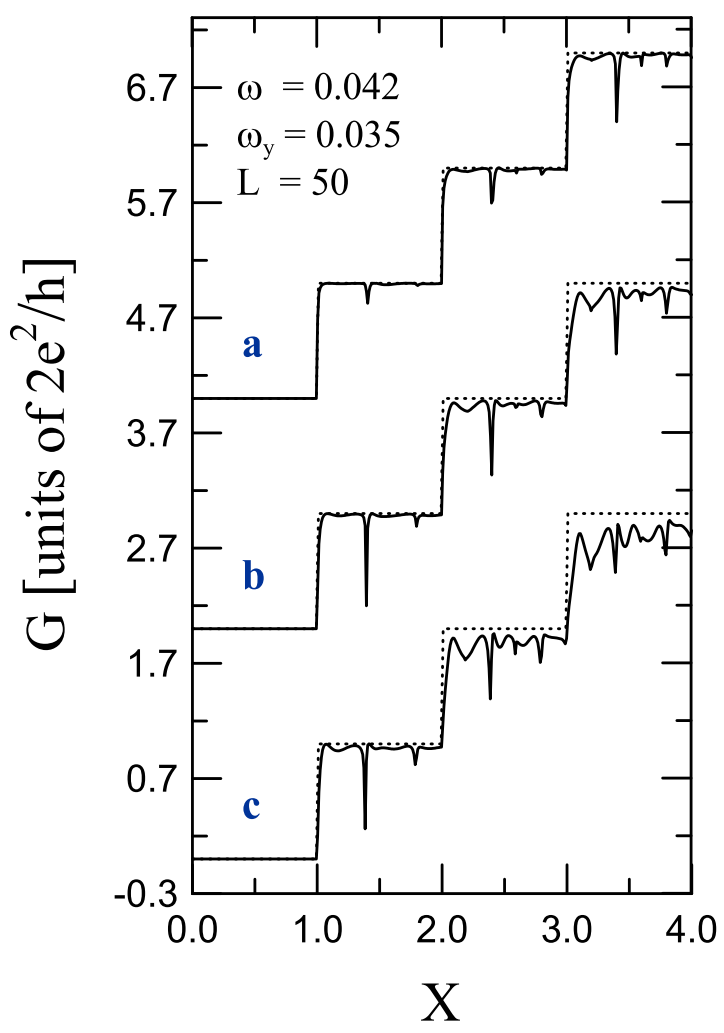

FIG. 3: Conductance $G$ as a function of $X$ for frequency $\omega=0.042(\simeq 0.6 \Delta \varepsilon)$, with time-modulated range $L=50$. The amplitude of the electric field are $\mathcal{E}_{0}=\mathrm{a}, 0.002(\simeq 22.6$ $\mathrm{V} / \mathrm{cm}) ; \mathrm{b}, 0.003(\simeq 33.9 \mathrm{~V} / \mathrm{cm}) ;$ and c, $0.004(\simeq 45.2 \mathrm{~V} / \mathrm{cm})$. The curves are vertically offset for clarity.

fix the length $L$ of the time-modulated region while varying the field strength $\mathcal{E}_{0}$, in which the angular frequencies are chosen to be $\omega=0.028(\nu=\omega / 2 \pi \cong 61 \mathrm{GHz})$ and $0.042(\nu \cong 91 \mathrm{GHz})$, as depicted in Figs. 4 and 3 respectively. The $L$ in both the figures are chosen to be $L=50(\simeq 0.4 \mu \mathrm{m})$. The $G$ characteristics are represented by the dependence on $X$, the suitably rescaled chemical potential $\mu$. According to this scale, when $\mu$ is changed by a subband energy level spacing $\Delta \varepsilon$, it corresponds to $\Delta X=1$, and when $\mu$ is changed by $\hbar \omega$, it corresponds to $\Delta X=\omega / \Delta \varepsilon=0.4$ and 0.6 for Figs. 4 and 3 respectively. In addition, when $X=N, \mu$ is at the $N$-th subband bottom, namely $N=1,2$, or 3 in these figures.

In our numerical examples, the narrow constriction is chosen to be that in a high mobility GaAs $-\mathrm{Al}_{x} \mathrm{Ga}_{1-x} \mathrm{As}$ with a typical electron density $n \sim 2.5 \times 10^{11} \mathrm{~cm}^{-2}$, and $m^{*}=0.067 m_{e}$. Correspondingly, our choice of length unit $a^{*}=1 / k_{\mathrm{F}}=79.6 \AA$, energy unit $E^{*}=$ $\hbar^{2} k_{\mathrm{F}}^{2} /\left(2 m^{*}\right)=9 \mathrm{meV}$, and frequency unit $\omega^{*}=E^{*} / \hbar=$ $13.6 \mathrm{THz}$. We also choose $\omega_{y}=0.035$ such that the transverse energy level spacing $\Delta \varepsilon=2 \omega_{y}=0.07$, and the effective narrow constriction width is of the order of $0.1 \mu \mathrm{m}$. In the following, in presenting the dependence of $G$ on $\mu$, it is more convenient to plot $G$ as a function 


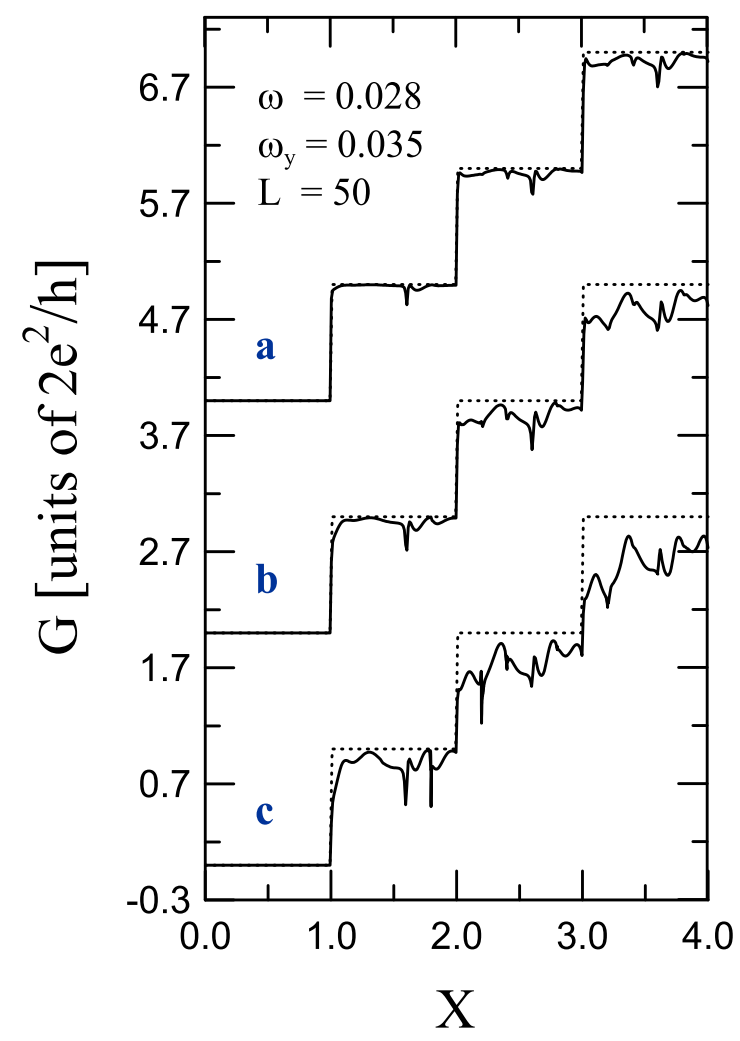

FIG. 4: Conductance $G$ as a function of $X$ for frequency $\omega=0.028(\simeq 0.4 \Delta \varepsilon)$, with time-modulated range $L=50$. The amplitude of the electric field are $\mathcal{E}_{0}=$ a, $0.002(\simeq 22.6$ $\mathrm{V} / \mathrm{cm}) ; \mathrm{b}, 0.003(\simeq 33.9 \mathrm{~V} / \mathrm{cm}) ;$ and $\mathrm{c}, 0.004(\simeq 45.2 \mathrm{~V} / \mathrm{cm})$. The curves are vertically offset for clarity.

of $X$ instead, where

$$
X=\frac{\mu}{\Delta \varepsilon}+\frac{1}{2} .
$$

With this conversion, $X$ is in units of $\Delta \varepsilon$, and the integral value of $X$ is the number of propagating channels through the NC.

In Fig. [4 the field amplitudes are chosen to be $\mathcal{E}_{0}=$

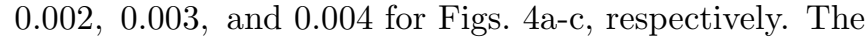
angular frequency $\omega$ is chosen to be 0.028 , whose energy interval $\omega$ corresponds to an interval $\Delta X=\omega / \Delta \varepsilon=0.4$. The dotted curves are the unperturbed results. In general, we find the suppressed features in $G$ that escalate with both the chemical potential and $\mathcal{E}_{0}$, as depicted in Figs. 目-c. Besides, there are dip structures in these figures, which can be understood to be the formation of QBS's due to time-modulated fields ${ }^{26.33 .34 .35}$ These QBS's, formed at energies near the threshold of subbands, trap temporarily conduction electrons and give rise to drops in $G$. However, the trapped electron can also be excited out of the QBS, resulting a smaller $G$ reduction: $|G|<1$, in units of $2 e^{2} / h^{26}$ In contrast, the impurity-induced dips are the results of merely elastic scattering and thus have $G$ reduction $|G|=1 \stackrel{8.9}{\text { It }}$ is shown when we increase the $\mathcal{E}_{0}$, these dip structures be- come more deeper and shift slightly toward the lower energy direction. Our results demonstrate the manifestation of a new, and time-dependent electric-field-induced QBS formed from transitions due to coherent inelastic scatterings.

Figures 3a-c have common types of dip structures. First, the dip structures are found at around $X=1.6,2.6$, and 3.6, that is, at $\Delta X=0.4$ beneath a subband bottom. These dip structures are due to the processes that an electron in the $N$ th subband, and at energy $(N+1)-\Delta X$, can absorb an energy $\hbar \omega$ and become bounded in the QBS just beneath the threshold of the $(N+1)$ th subband. In other words, this process is $(\Delta n, \Delta m)=(+1,+1)$. Second, the small dip structures at around $X=2.4$ and 3.4 are attributed to the processes that an electron in the $N$ th subband, and at energy $N+\Delta X$, can give away $\hbar \omega$ to be trapped temporarily in the $(N+1)$ th subband bottom. This process corresponds to $(\Delta n, \Delta m)=(+1,-1)$. Third, the dips at around $X=2.2$ and 3.2 are contributed by two different kinds of transitions, that is, $(+2,+2)$ and $(-1,+3)$ processes. The former corresponds to that an electron in the $N$ th subband with energy $(N+2)-2 \Delta X$ can absorb $2 \hbar \omega$ to the $(N+2)$ th subband bottom, and the latter corresponds to those make transitions from the $N$ th subband, with energy $(N-1)+3 \Delta X$, to the $(N-1)$ th subband bottom. We should point out that all the above are intersubband and intersideband transitions.

Moreover, in Figs. 40 and c, there are dip structures at around $X=3.0$ and 4.0 , which correspond to $(+2,0)$ transitions. These structures are due to intersubband and intrasideband transitions, namely that the electron energy remains unchanged. Besides, in Fig. 四, there are dips at around $X=1.8,2.8$, and 3.8, which correspond to $(0,-2)$ transitions. We can see that these multi-photon transitions manifest only when the applied field intensity is larger.

In Fig. 31 the field amplitudes are chosen to be the same as Fig. 4. The angular frequency $\omega$ is chosen to be 0.042 , whose energy interval $\omega$ corresponds to an interval $\Delta X=\omega / \Delta \varepsilon=0.6$. There are dip structures in these figures associated with the formation of time-depdent fieldinduced QBS's ${ }^{26.33 .34 .35}$ There are common types of dip structures in Figs. Ba-c. First, the dip structures are found at around $X=1.4,2.4$, and 3.4 , which are associated with $(+1,+1)$ processes. These dip structures correspond to that an electron in the $N$ th subband, and at energy $(N+1)-\Delta X$, can absorb an energy $\hbar \omega$ and become bounded as a QBS in the $(N+1)$ th subband. Second, the small dips at around $X=2.6$ and 3.6, associated with $(+1,-1)$ processes, correspond to that an electron in the $N$ th subband with energy $N+\Delta X$ can give away $\hbar \omega$ to be trapped temporarily in the $(N+1)$ th subband bottom. Third, the dips at around $X=1.8$, 2.8 , and 3.8 , associated with $(+2,+2)$ processes, correspond to that an electron in the $N$ th subband, at energy $(N+2)-2 \Delta$, can absorb $2 \hbar \omega$ to the $(N+2)$ th subband bottom. Moreover, in Figs. 3b and c, dip structures at 
around $X=3.0$ and 4.0 are identified to be $(+2,0)$ intersubband and intrasideband transitions. Furthermore, in Fig. 3r, there are dips at around $X=2.2$ and 3.2, which are associated with $(0,-2)$ transitions.

As mentioned in our previous work,, 30 to observe the above predicted effects, the experimental setup needs to fulfill two requirements. First, the bolometric effect due to the absorption of photons in the QPC's end-electrodes has to be suppressed or totally eliminated. Recent experiments show that the transport characteristics are masked by the bolometric effect when the entire QPC, including the end-electrodes, is exposed to the incident electromagnetic field $r^{20}$ Second, the length $L$ of the time-dependent field has to be shorter than the wave length of the incident field. The purpose is to increase the coupling between the electrons and the photons by breaking the longitudinal translational invariance. That the coupling between the photon field and the conduction electrons can be much enhanced, when either the electrons are confined or the time-dependent field has a localized profile, has been pointed out recently by Yakubo et al ${ }^{39}$ Thus the QPC needs to be in the near-field regime of the timedependent field.

To avoid the bolometric effect, we suggest to apply ac field to the split gates of the QPC instead of shining an electromagnetic wave upon the QPC. The split gates are negatively biased with respect to a common ground, and made of superconducting materials with superconducting wires connecting to an ac-signal generator. This generator can be available using the IMPATT diode that has successfully been demonstrated to cover the complete millimeter range $(30-300 \mathrm{GHz}) \stackrel{40}{4}$ This proposed experimental setup is expected to generate a transversely polarized electric field only in the narrow constriction region while keeping the two-end electrodes from the time-modulated field. In this work, though the time-dependent region covers only part of the narrow constriction, we believe these two situations will manifest similar features. Given the availability of millimeter wave sources, $\stackrel{40}{,}$ the suggested experimental setup would be manageable by the present nanotechnology. The features reported in this work, however, are not limited to millimeter waves.

\section{CONCLUSION}

A generalized scattering-matrix method has been developed for investigating coherent quantum transport in narrow constrictions with a transversely polarized timedependent electric field. This method allows us to solve nonpertubatively the time-dependent Schrödinger equation in the numerical sense. Since the energy conservation law is violated in such a time-modulated system, a conventional transfer-matrix method technique is inapplicable. Using the present numerical method, not only the transmission and reflection probabilities of systems can be calculated, all the subband and sideband states can be obtained.

The scattering processes due to the time-dependent external field are both inelastic and coherent. Since this field is transversely polarized, electrons can make both intersubband and intersideband transitions. This increases the complexity in calculation, but has more interesting features. Different dip structures associated with different intersubband and intersideband transitions to the vicinity of a subband bottom are found. These dip structures can be understood as the formation of a QBS at energy near a subband bottom due to its singular DOS. ${ }^{26}$ Moreover, due to the tunability of frequency and intensity of the field, this proposed configuration can be applied to be a high-frequency detector. We expect that these dip structures could also be found when the QPC has a varying width. We hope that the present method will be utilized to study new transport phenomena in mesoscopic nanostructures.

\section{Acknowledgments}

The authors would like to thank the National Science Council of the Republic of China for financially supporting this research under Contract No. NSC88-2112-M009-028. Computational facilities supported by the National Center for High-performance Computing are gratefully acknowledged.
1 D.K. Ferry and S.M. Goodnick, Transport in Nanostructures (Cambridge University Press, Cambridge, U.K., 1997).

2 Y. Imry, Introduction to Mesoscopic Physics (Oxford University Press, Oxford, U.K., 1997).

3 B.J. van Wees, H. van Houton, C.W.J. Beenakker, J.G. Williamson, L.P. Kouwenhoven, D. van der Marel, and C.T. Foxon, Phys. Rev. Lett. 60, 848 (1988).

4 D.A. Wharam, T.J. Thornton, R. Newbury, M. Pepper, H. Ahmed, J.E.F. Frost, D.G. Hasko, D.C. Peacock, D.A. Ritchie, and G.A.C. Jones, J. Phys. C 21, L209 (1988).

5 B.J. van Wees, L.P. Kouwenhoven, H. van Houton, C.W.J.
Beenakker, J.E. Mooji, C.T. Foxon, and J.J. Harris, Phys. Rev. B 38, 3625 (1988).

${ }^{6}$ L.I. Glazman, G.B. Lesovik, D.E. Khmelnitskii, and R.I. Shekhter, Pis'ma Zh. Eksp. Teor. Fiz. 48, 329 (1988) [JETP Lett. 48, 238 (1988)].

7 L. I. Glazman and M. Jonson, Phys. Rev. B 41, 10686 (1990).

8 C.S. Chu and R.S. Sorbello, Phys. Rev. B 40, 5941 (1989).

9 P.F. Bagwell, Phys. Rev. B 41, 10354 (1990).

10 E. Tekman and S. Ciraci, Phys. Rev. B 43, 7145 (1991).

11 J.A. Nixon, J.H. Davies, and H.U. Baranger, Phys. Rev. B 43, 12638 (1991). 
12 Y.B. Levinson, M.I. Lubin, and E.V. Sukhorukov, Phys. Rev. B 45, 11936 (1992).

13 Y. Takagaki and D.K. Ferry, Phys. Rev. B 46, 15218 (1992).

14 Ch. Kunze and R. Lenk, Solid State Commun. 84, 457 (1992).

15 F. Hekking and Y.V. Nazarov, Phys. Rev. B 44, 11506 (1991).

16 Q. Hu, Appl. Phys. Lett. 62, 837 (1993).

17 R.A. Wyss, C.C. Eugster, J.A. del Alamo, and Q. Hu, Appl. Phys. Lett. 63, 1522 (1993).

18 R.A. Wyss, C.C. Eugster, J.A. del Alamo, and Q. Hu, Appl. Phys. Lett. 66, 1144 (1995).

19 Q. Hu, S. Verghese, R.A. Wyss, Th. Schäpers, J. del Alamo, S. Feng, Y. Yakubo, M.J. Rooks, M.R. Melloch, and A. Förster, Semicond. Sci. Technol. 11, 1888 (1996).

20 J.A. del Alamo, C.C. Eugster, Q. Hu, M.R. Melloch, and M.J. Rooks, Superlatt. and Microstru. 23, 121 (1998).

21 L. Fedichkin, V. Ryzhii, and V. V'yurkov, J. Phys. Condens. Matter 5, 6091 (1993).

${ }^{22}$ T.J.B.M. Janssen, J.C. Maan, J. Singleton, N.K. Patel, M. Pepper, J.E.F. Frost, D.A. Ritchie, and G.A.C. Jones, J. Phys. Condens. Matter 6, L163 (1994).

${ }^{23}$ L.Y. Gorelik, A. Grincwajg, V.Z. Kleiner, R.I. Shekhter, and M. Jonson, Phys. Rev. Lett. 73, 2260 (1994).

24 A. Grincwajg, L.Y. Gorelik, V.Z. Kleiner, and R.I.
Shekhter, Phys. Rev. B 52, 12168 (1995).

25 F.A. Maaø and L.Y. Gorelik, Phys. Rev. B 53, 15885 (1996).

26 C.S. Chu and C.S. Tang, Solid State Commun. 97, 119 (1996).

27 Ola Tageman, L.Y. Gorelik, R.I. Shekter, and M. Jonson, J. Appl. Phys. 81, 285 (1996).

28 Ola Tageman and L.Y. Gorelik, J. Appl. Phys. 83, 1513 (1997).

29 S. Feng and Q. Hu, Phys. Rev. B 48, 5354 (1993).

30 C.S. Tang and C.S. Chu, Phys. Rev. B 60, 1830 (1999).

31 R. Landauer, IBM J. Res. Dev. 1,223 (1957).

32 M. Büttiker, Phys. Rev. Lett. 57, 1761 (1986).

33 P.F. Bagwell and R.K. Lake, Phys. Rev. B 46, 15329 (1992).

34 C.S. Tang and C.S. Chu, Phys. Rev. B 53, 4838 (1996).

35 C.S. Tang and C.S. Chu, Physica B 254, 178 (1998).

36 M. Büttiker, Phys. Rev. B 41, 7906 (1990).

37 D.D. Coon and H.C. Liu, J. Appl. Phys. 58, 2230 (1985).

38 D.Y.K. Ko and J.C. Inkson, Phys. Rev. B 38, 9945 (1988).

39 K. Yakubo, S. Feng, and Q. Hu, Phys. Rev. B 54, 7987 (1996).

40 See, for example, P. Bhartia and I.J. Bahl, Millimeter Wave Engineering and Applications (John Wiley \& Sons, New York, 1984), Chap. 3. 